Supporting information

\title{
Modulating the Electrode-Electrolyte Interface with Cationic Surfactants in Carbon Dioxide
} Reduction

\author{
Soumyodip Banerjee, ${ }^{1}$ Xu Han, ${ }^{1}$ and V. Sara Thoi ${ }^{1 *}$ \\ ${ }^{1}$ Department of Chemistry, Johns Hopkin University, Baltimore, MD, USA. \\ *corresponding author: sarathoi@jhu.edu
}

\section{Table of Content}

\section{Experimental Details}

Figure S1. Electrolysis results at different electrolyte purity.

Figure S2. Bulk electrolyte $\mathrm{pH}$ with subsequent addition of CTAB

Figure S3. Equivalent circuit diagram for EIS simulations

Figure S4. Fitting of experimental Nyquist plots.

Figure S5. Extracted electrolyte resistance from Nyquist plots.

Figure S6. SEM image of $\mathrm{Cu}$ foil.

Figure S7. Specific capacitance and surface roughness factor determination of $\mathrm{Cu}$ foil.

Figure S8. Linear sweep voltammetry in phosphate buffer solution.

Figure S9. Total Faradaic efficiency for electrolysis carried out at different potentials.

Figure S10. Stability analysis for electrolysis utilizing $\mathrm{Cu}$ foil in presence and absence of CTAB

Figure S11. $\mathrm{CO}_{2} \mathrm{RR}$ current density and faradaic efficiency in presence of $\mathrm{NaBr}$.

Figure S12. $\mathrm{CO}_{2} \mathrm{RR}$ Faradaic efficiency in absence and presence of CTAB and CTAP

Figure S13. Controlled potential electrolysis using CTAB and sodium oleate at $-0.9 \mathrm{~V}$ vs RHE.

Figure S14. Charge transfer resistance vs. polarization potential in the presence and absence of CTAB.

Figure S15. HER and $\mathrm{CO}_{2} \mathrm{RR}$ current density at different $\mathrm{CTAB}$ concentrations.

Figure S16. Current density and charge transfer resistance plots in the presence of surfactants comprising different chain lengths. 


\section{Experimental Details}

Synthesis of (1-hexyl)trimethylammonium perchlorate (CTAP). (1-Hexyl)trimethylammonium perchlorate was synthesized using an anion exchange method reported in literature. ${ }^{1}$ An aqueous solution of CTAB was added dropwise in excess $70 \%$ perchloric acid. A white precipitate formed and was filtered and washed with cold DI until the $\mathrm{pH}$ of the water wash is neutral. The precipitate was washed with acetone and dried overnight under air. The IR spectrum of synthesized CTAP matches the spectrum found on the AIST Spectral Database for Organic Compounds.

Electrochemical Impedance Spectroscopy. ElS experiments carried out in $\mathrm{N}_{2}$ and $\mathrm{CO}_{2}$ saturated $\mathrm{NaHCO}_{3}$ solution in the presence of $67 \mu \mathrm{M} \mathrm{CTAB}$. The first semicircle feature in Nyquist plots does not change with polarization potential whereas the shape of $2^{\text {nd }}$ semicircle is influenced by applied polarization potential. The $2^{\text {nd }}$ semicircle has been simulated to probe charge transfer processes and structural changes in the double layer in the presence of CTAB. We used the equivalent circuit in Figure S3 to simulate the experimental datapoints.

Double layer capacitance value has been calculated using the formula, where $\mathrm{C}_{\mathrm{dl}}$ is the double layer capacitance, $\mathrm{R}_{\mathrm{CT}}$ is the charge transfer resistance, $\mathrm{CPE}$ is the constant phase element, and the value of $\mathrm{N}$ mainly depends on surface roughness which is also achieved by simulation of experimental Nyquist plots.

$$
\mathrm{C}_{\mathrm{dl}}=\left\{\mathrm{R}_{\mathrm{CT}}^{(1-\mathrm{N})} \mathrm{CPE}\right\}^{(1 / \mathrm{N})}
$$


(a)

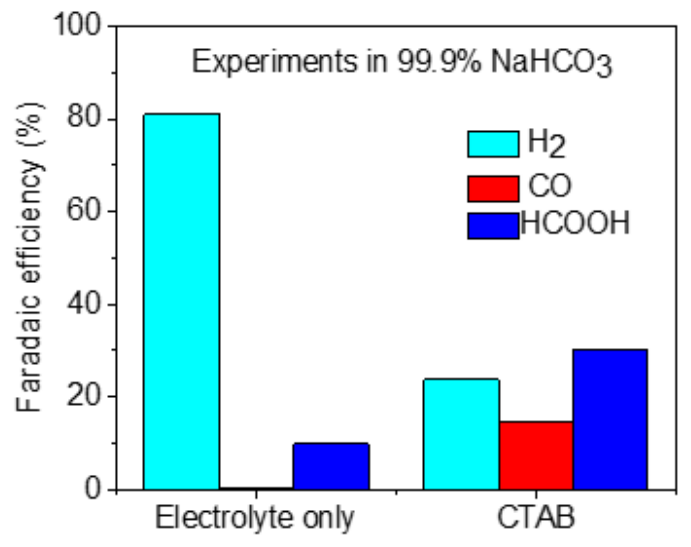

(b)

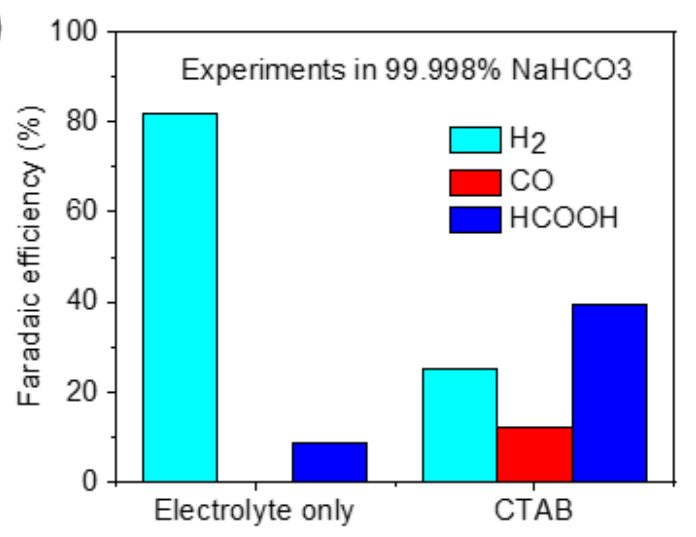

Figure S1. Faradaic efficiency at $-0.7 \mathrm{~V}$ in the absence and presence of $67 \mu \mathrm{M}$ CTAB in electrolyte prepared with as-purchased (a) $99.9 \%$ pure $\mathrm{NaHCO}_{3}$ and (b) $99.998 \% \mathrm{NaHCO}_{3}$ ).



Figure. S2. Bulk electrolyte $\mathrm{pH}$ with subsequent addition of CTAB in $0.1 \mathrm{M} \mathrm{NaHCO}_{3}$, demonstrating minimal changes to $\mathrm{CO}_{2}$ solubility with increasing surfactant concentration. 


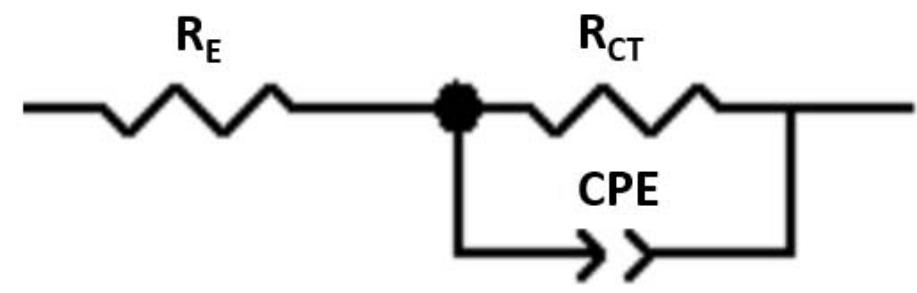

Figure S3. Equivalent circuit diagram for simulation of experimental Nyquist plots.
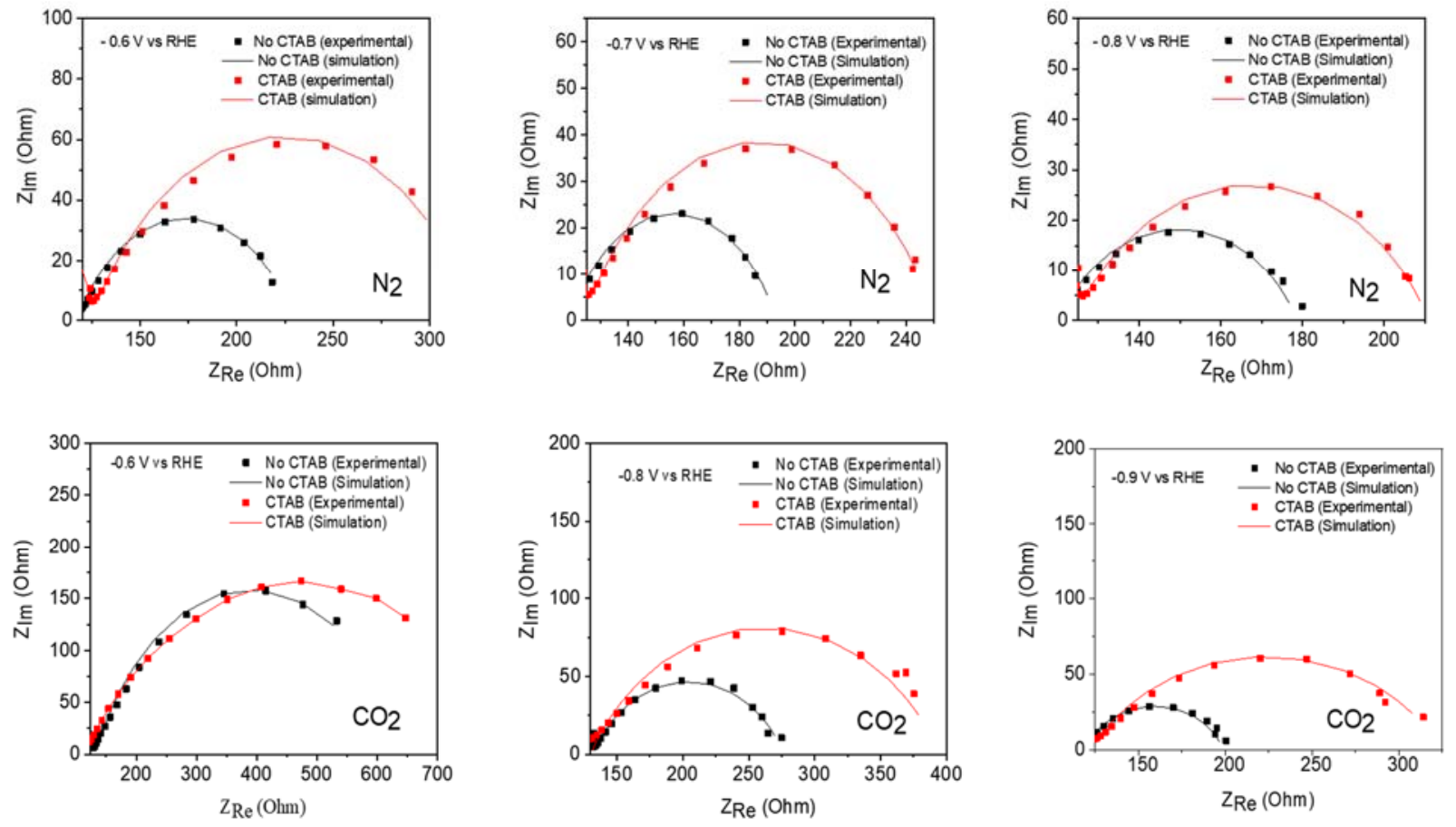

Figure S4. Nyquist plot (experimental and simulation) for EIS experiments carried out in $\mathrm{N}_{2}$ and $\mathrm{CO}_{2}$ saturated $0.1 \mathrm{M} \mathrm{NaHCO}_{3}$ solution in the presence and absence of $67 \mu \mathrm{M}$ CTAB. 

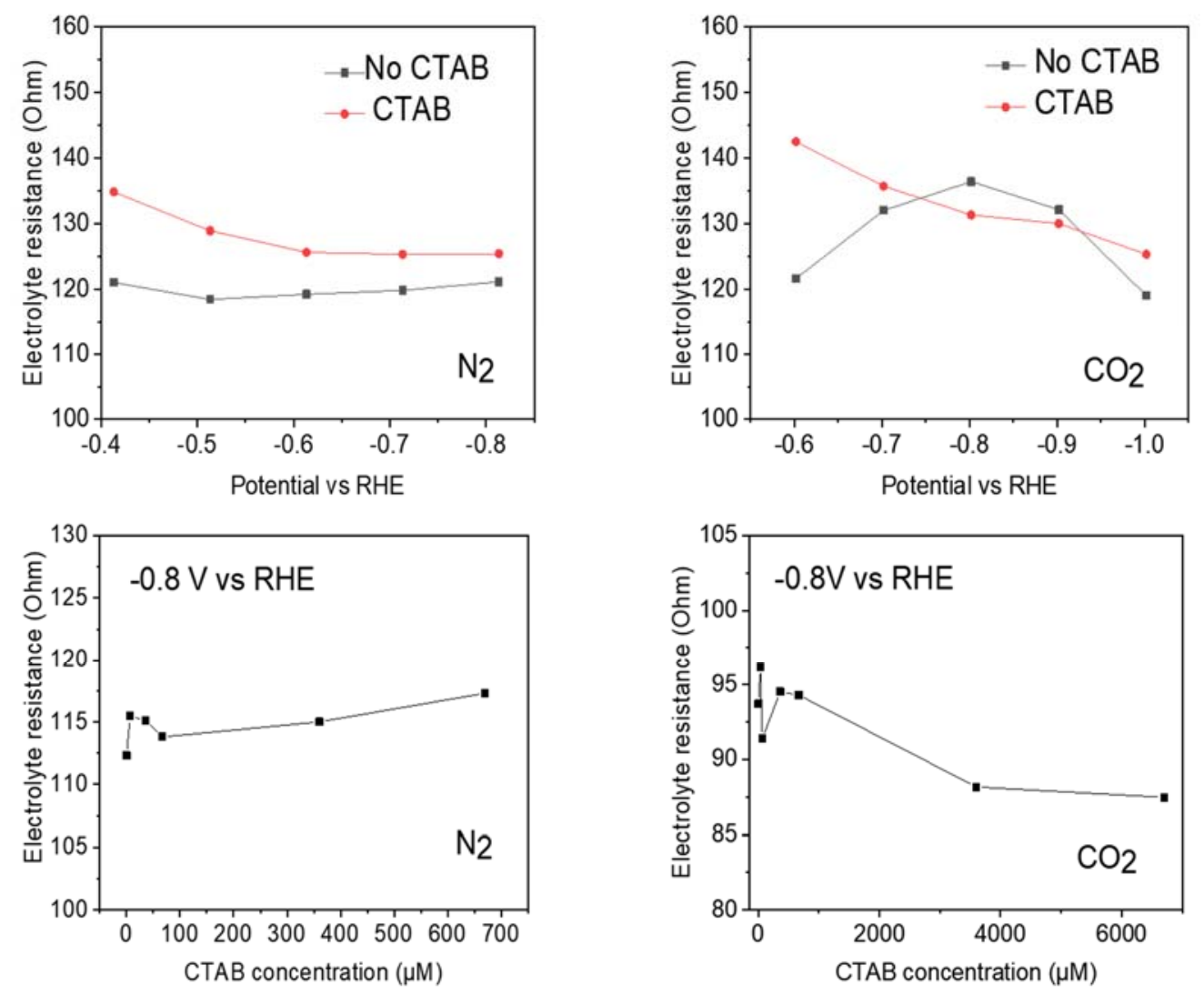

Figure S5. Electrolyte resistance by modelling impedance experiments in $\mathrm{N}_{2}$ and $\mathrm{CO}_{2}$ atmospheres in the presence and absence of $67 \mu \mathrm{M} \mathrm{CTAB}$ (top) and at different CTAB concentrations (bottom).

(a)



(b)



(c)

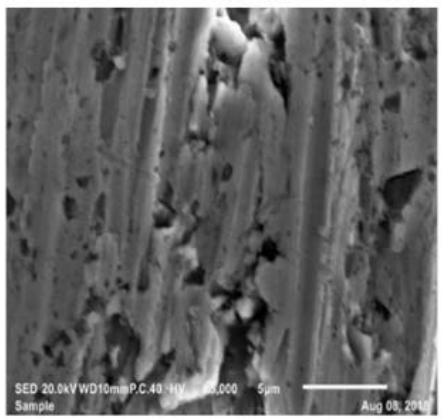

Figure S6. SEM images of the (a) mechanically polished $\mathrm{Cu}$ foil before electrolysis, and the $\mathrm{Cu}$ foil after a 2-h electrolysis at - $0.8 \mathrm{~V}$ vs RHE in the (b) absence and (c) presence of $67 \mu \mathrm{M} \mathrm{CTAB}$ in $\mathrm{CO}_{2}$-saturated $0.1 \mathrm{M} \mathrm{NaHCO}_{3}$ solution. 


\section{Electrode surface roughness factor:}

Electrochemical double layer capacitance was measured by using cyclic voltammetry at different scan rate in $0.1 \mathrm{M} \mathrm{NaHCO}_{3}$ solution saturated with $\mathrm{CO}_{2}$ in the presence of $67 \mu \mathrm{M}$ CTAB to observe surface restructuring of the $\mathrm{Cu}$ foil after controlled potential electrolysis. The difference of cathodic and anodic current has been plotted with scan rate and the slope has been calculated. The similar slopes before and after bulk electrolysis indicate minimal changes to the surface structure, corroborating SEM analysis.

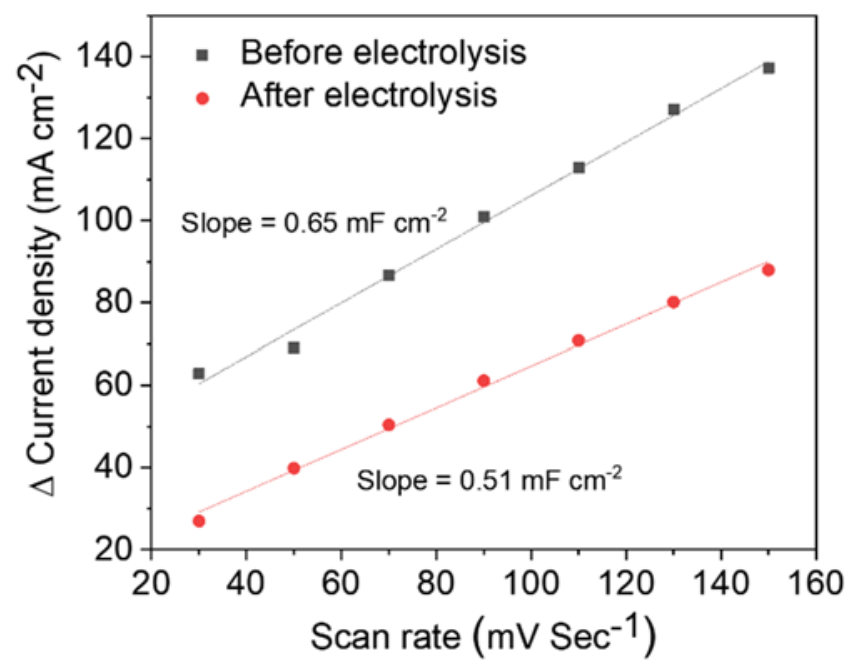

Figure S7. Plot of $\left(i_{\text {cathodic }}-i_{\text {anodic }}\right)$ with respect to scan rate for cyclic voltammetry carried out with $\mathrm{Cu}$ foil working electrode before and after bulk electrolysis.

(a)

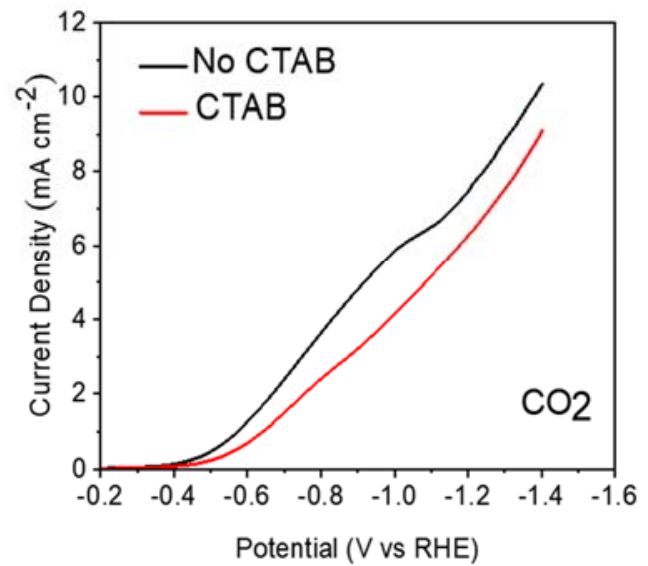

(b)

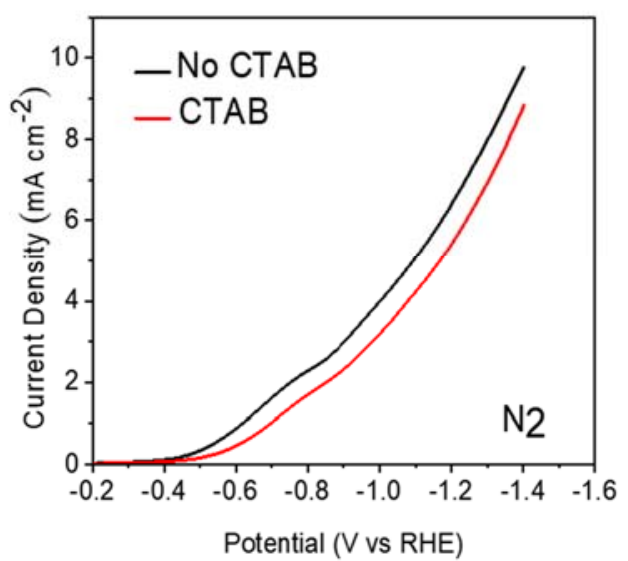

Figure S8. LSV in the absence (black) and presence of $67 \mu \mathrm{M} \mathrm{CTAB}$ (red) under (a) $\mathrm{CO}_{2}$ and (b) $\mathrm{N}_{2}$ in 0.1 M phosphate buffer ( $\mathrm{pH} 6.8)$. 




Figure S9. Total Faradaic Efficiency at all tested potentials in the absence and presence of CTAB in $0.1 \mathrm{M}$ $\mathrm{NaHCO}_{3}$ solutions. 
(a)

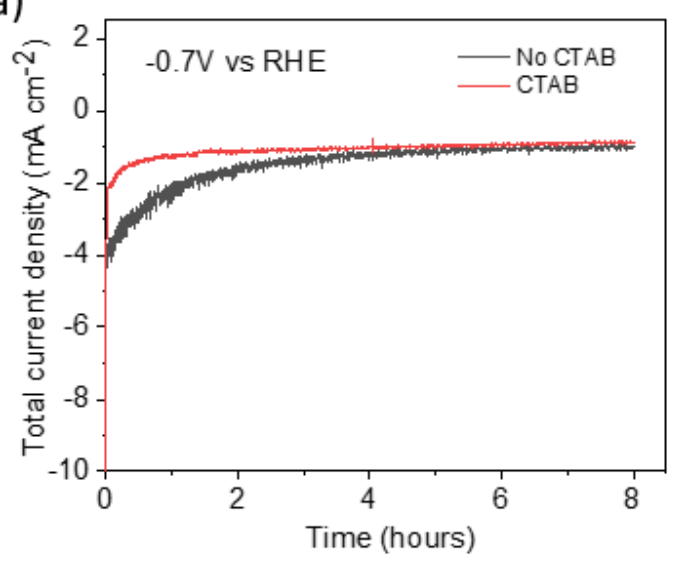

(b)

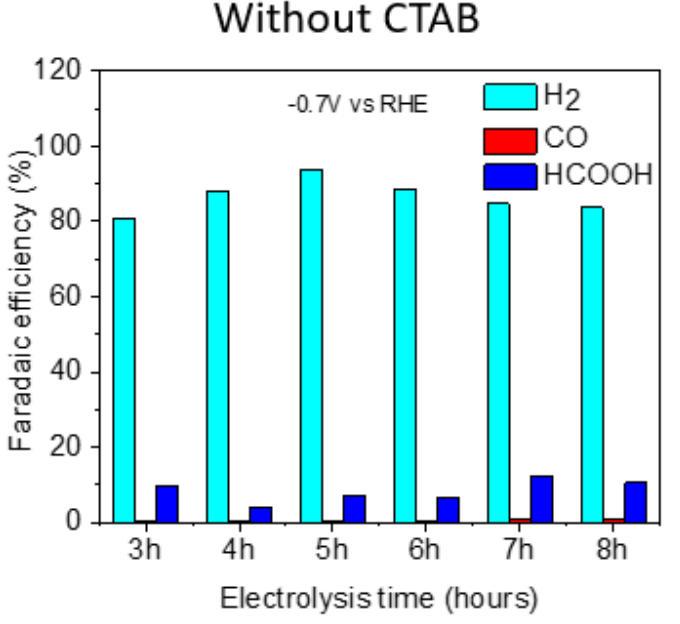

With CTAB

(c)

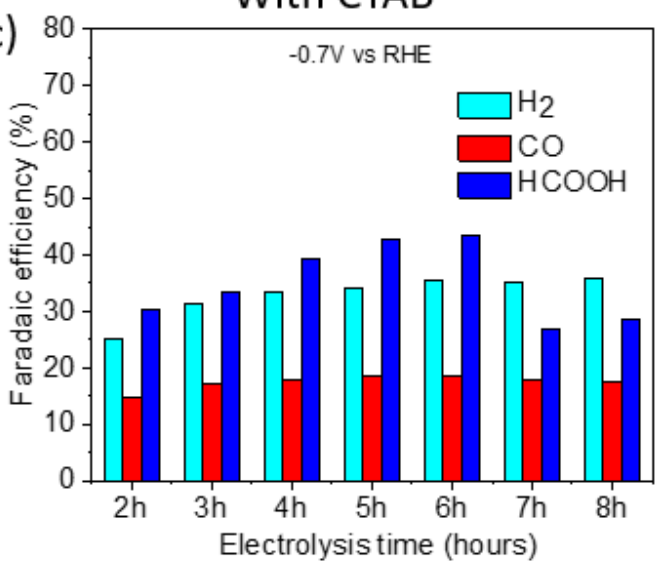

Figure S10. (a) Total Current density and Faradaic efficiency at $-0.7 \mathrm{~V}$ in $\mathrm{CO}_{2}$-saturated $0.1 \mathrm{M} \mathrm{NaHCO}_{3}$ solution (b) in the absence and (c) in the presence of $67 \mu \mathrm{M}$ of CTAB for long term electrolysis over 8 hours. 
(a)

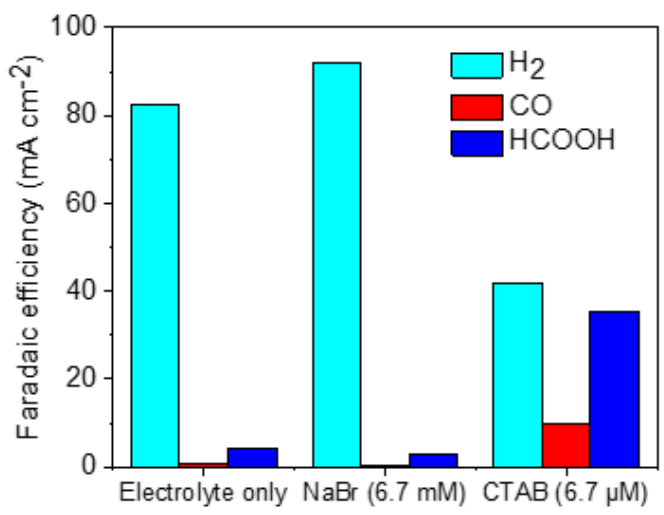

(b)

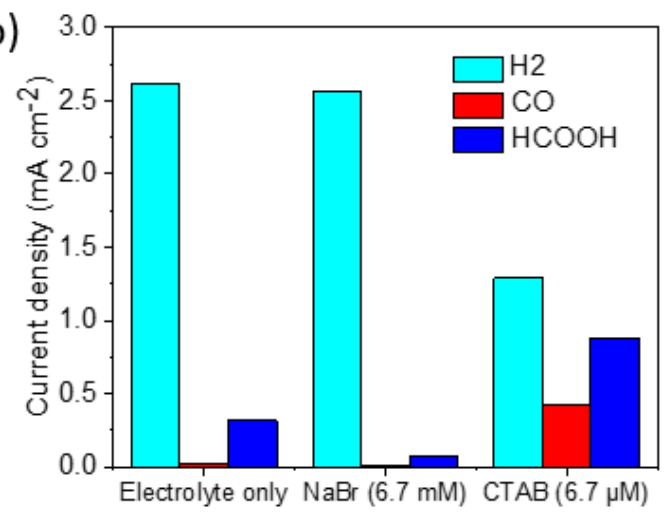

Figure S11. (a) Total current density and (b) Faradaic efficiency at $-0.8 \mathrm{~V}$ in $\mathrm{CO}_{2}$-saturated $0.1 \mathrm{M} \mathrm{NaHCO}_{3}$ solution in the absence and in the presence of $6.7 \mu \mathrm{M} \mathrm{NaBr}$ and $6.7 \mu \mathrm{M} \mathrm{CTAB}$, demonstrating that halide adsorption does not play a role in the observed $\mathrm{CO}_{2}$ selectivity.

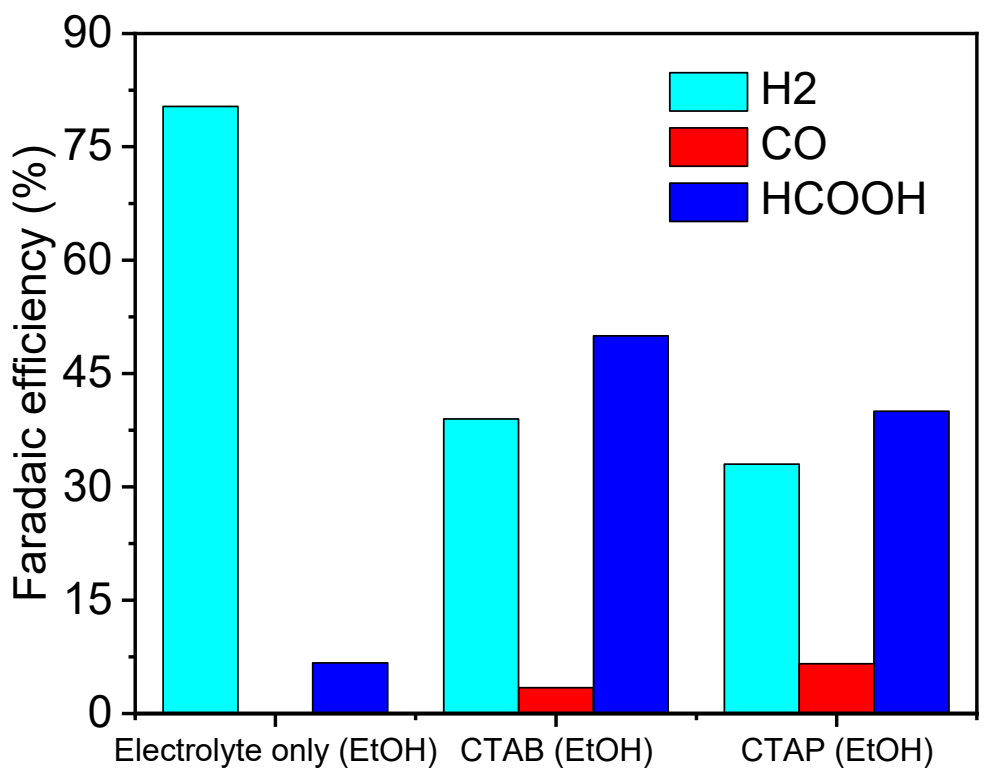

Figure S12. Faradaic efficiency at $-0.8 \mathrm{~V}$ in $\mathrm{CO}_{2}$-saturated $0.1 \mathrm{M} \mathrm{NaHCO}_{3}$ solution in the absence and presence of $6.7 \mu \mathrm{M}$ CTAB and $6.7 \mu \mathrm{M}$ CTAP. Since CTAP is not soluble in water, the white precipitate was first dissolved in ethanol and $50 \mu \mathrm{L}$ was added to the $40 \mathrm{~mL}$ electrolyte solution. For direct comparison, the CTAB and electrolyte-only solutions also contain the same amount of ethanol. 




Figure S13. Faradic efficiency at $-0.9 \mathrm{~V}$ in $\mathrm{CO}_{2}$-saturated $0.1 \mathrm{M} \mathrm{NaHCO}_{3}$ solution in the absence and presence of $67 \mu \mathrm{M}$ CTAB and sodium oleate.

(a)



(b)

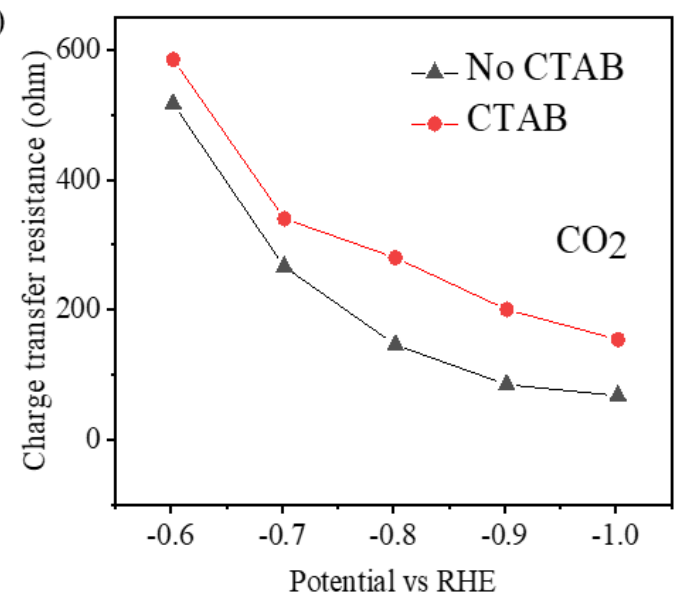

Figure S14. Charge transfer resistance by modeling impedance experiments in (a) $\mathrm{N}_{2}$-purged, (b) $\mathrm{CO}_{2}$ purged $0.1 \mathrm{M} \mathrm{NaHCO}_{3}$ solution in the presence (red circle) and absence (black triangle) of CTAB 




Figure S15. Current density results of bulk electrolysis carried out at $-0.8 \mathrm{~V}$ in $\mathrm{CO}_{2}$-saturated $0.1 \mathrm{M}$ $\mathrm{NaHCO}_{3}$ solution at different CTAB concentration.


Figure S16. (a) HER and $\mathrm{CO}_{2} \mathrm{RR}$ current density for controlled potential electrolysis and (b) charge transfer resistance extracted in $\mathrm{CO}_{2}$-purged $0.1 \mathrm{M} \mathrm{NaHCO}_{3}$ solution in the presence of $67 \mu \mathrm{M}$ cationic surfactants comprising different numbers of carbon atom in the main alkyl chain. 


\section{References.}

1. Goga, S. T.; Glazkova, E. N.; Mchedlov-Petrosyan, N. O. The Thermodynamic Characteristics of Dissolution and Solvation of Cetyltrimethylammonium Perchlorate in the Water-Propan-2-Ol System. Russ. J. Phys. Chem. A 2010, 82, 1451-1455. https://doi.org/10.1134/s0036024408090070. 